\title{
THE FUKUSHIMA INVERSE PROBLEM
}

\author{
Marta Martinez-Camara, Ivan Dokmanić, \\ Juri Ranieri, Robin Scheibler and Martin Vetterli \\ School of Computer and Communication Sciences, \\ Ecole Polytechnique Fédérale de Lausanne (EPFL), \\ CH-1015 Lausanne, Switzerland, \\ firstname.familyname@epfl.ch
}

\begin{abstract}
Knowing what amount of radioactive material was released from Fukushima in March 2011 is crucial to understand the scope of the consequences. Moreover, it could be used in forward simulations to obtain accurate maps of deposition. But these data are often not publicly available, or are of questionable quality. We propose to estimate the emission waveforms by solving an inverse problem. Previous approaches rely on a detailed expert guess of how the releases appeared, and they produce a solution strongly biased by this guess. If we plant a nonexistent peak in the guess, the solution also exhibits a nonexistent peak. We propose a method based on sparse regularization that solves the Fukushima inverse problem blindly. Together with the atmospheric dispersion models and worldwide radioactivity measurements our method correctly reconstructs the times of major events during the accident, and gives plausible estimates of the released quantities of Xenon.
\end{abstract}

Index Terms - Fukushima, Daiichi, nuclear power plant, explosion, inverse problems, FLEXPART, dispersion

\section{INTRODUCTION}

Nuclear power plants (NPP) provide an abundant, relatively cheap, and carbon-neutral source of energy. However, they also introduce a possibility, albeit a very remote one, of a major accident. A nuclear accident is defined by the International Atomic Energy Agency as an event having lethal consequences, environmental effects such as large radioactivity releases, and producing long-lasting facility defects such as core melts. Level 7 on the International Nuclear Events Scale is defined as a major release of radioactive material with widespread health and environmental effects requiring implementation of planned and extended countermeasures [1]. Two accidents have reached this level—Chernobyl in April 1986 and Fukushima Daiichi in March 2011.

The principal consequence of NPP accidents is the release of radioactive material. Transported through the atmosphere, it eventually gets widely spread, polluting the environment for centuries at a large scale. The exposure to the radioactive material causes cancer, teratogenesis, cognitive decline, and heart disease [2]. Thus it is imperative to monitor the radioactive contamination of soil, waters and atmosphere. Unfortunately, the contamination can only be accurately measured at a limited number of survey sites due to the cost

This work was supported by a doctoral fellowship from EPFL and an ERC Advanced Grant - Support for Frontier Research - SPARSAM Nr: 247006 .

\author{
Andreas Stohl \\ Norwegian Institute for Air Research, \\ (NILU), \\ 2027 Kjeller, Norway, \\ ast@nilu.no
}

of scientific grade equipment. This suggests the need for numerical simulations of atmospheric dispersion $[3,4]$.

But getting accurate concentration and deposition values through simulations requires the knowledge of the source term-we should know how much radioactive material was released at what times. Its precise estimate is essential to properly estimate the contamination and take risk reducing measures. However, these data are often not publicly available or it is unknown. In particular, some works $[4,5]$ challenge the data released by the Japanese government about the Fukushima accident. An option is to calculate the source term based on spatio-temporal samples of the concentration, that is, by solving an inverse problem.

We propose to estimate the source term by inverting the atmospheric dispersion. This would provide us with an estimate of how much radioactive material was released, and with a firm starting point for understanding the scope of the pollution through dispersion simulation. We summarize our effort as follows,

Problem 1 (Fukushima Inverse Problem). Given the measurements $\mathbf{y}$ collected at the survey sites, and the model $\mathbf{A}$, recover the temporal variation of the radioactive material release $\mathbf{x}$.

The main contribution of this paper is a solution to the Fukushima inverse problem formulated as a convex program. We use worldwide radioactivity measurements and weather data from March 2011 on. Unlike previous approaches, the proposed formulation correctly estimates the explosion and venting times based on Xenon emissions, without involving an expert-knowledge-based initial guess. The estimated release magnitudes match earlier expert estimates.

\section{MODELING AND PRIOR ART}

Numerical simulations relate the deposition at locations of interest with the amount of material emitted by the source. The link is established through atmospheric dispersion models. These models frequently assume a linear relationship,

$$
y(\xi, t)=\int_{0}^{t} \mathcal{A}(\xi, t, \tau) x(\tau) d \tau
$$

where $y(\xi, t)$ is the measured concentration of the material at the location $\xi \in \mathbb{R}^{2}$ at time $t, x(\tau)$ is the amount of material emitted at time $\tau$ and $\mathcal{A}(\xi, t, \tau)$ is the spatio-temporal kernel of atmospheric dispersion. In practice, we discretize the source term, and compute the deposition values at a finite number of locations and 
Solutions with Different A-priori Guesses

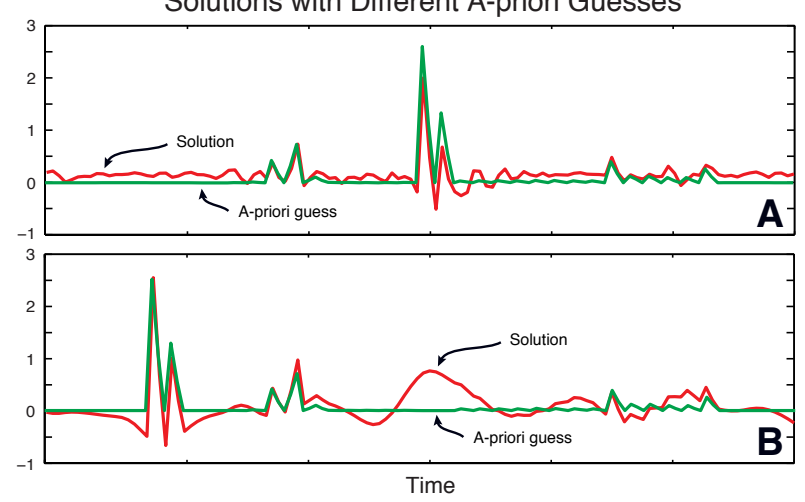

Fig. 1. Sensitivity of the formulation in [4] to initial guess. (A) Initial guess used in the paper and the corresponding reconstruction. (B) Modified initial guess with a relocated peak. The solution changes shape to match the new (in reality nonexistent) peak.

times. Therefore, the following discretized form is considered,

$$
\mathbf{y}=\mathbf{A x}
$$

where $\mathbf{y} \in \mathbb{R}^{m}$ contains the values of deposition at known locations in time and space and $\mathbf{x} \in \mathbb{R}^{n}$ describes the time-varying amount of material released by the source. $\mathbf{A} \in \mathbb{R}^{m \times n}$ is sometimes referred to as the sensitivity matrix, and computing it is a science in itself. Entries of $\mathbf{A}$ are complicated functions of the weather patterns, the type of the radioactive material, and the source position.

A popular class of models to compute $\mathbf{A}$ are the Lagrangian Dispersion Models (LDM). They model the particle trajectories by random walks, and then simulate the trajectories for a large number of particles. The entries of the sensitivity matrix are computed as empirical expectations of particle densities at given locations and times. We use an LDM called FLEXPART [6] since it is publicly available, has been validated against large-scale tracer experiments [7] and is considered reliable, being used for 15 years by a wide community. In principle, knowing the emission rates $\mathrm{x}$ and having the "correct" matrix $\mathbf{A}$ enable us to compute concentrations at arbitrary points in time and space.

Inverse problems have a rich theory. A good overview of linear inverse problems is given in [8]. Standard regularizations in the domain of atmospheric dispersion modeling involve smoothing based on the norm of the discretized second derivative of $\mathbf{x}$ [9]. There have also been attempts to solve the Fukushima Inverse Problem. The state-of-the-art approach is the one considered in [4]. The authors observe that regularization based only on the smoothed second derivative does not yield satisfactory results. They use a combination of measurement error, norm of the second derivative and an a priori guess of the emission pattern based on expert knowledge. However, the algorithm they propose requires a number of parameters, generally unknown, to be blindly estimated. Moreover, the obtained estimate tends to overfit the a priori guess, while being less sensitive to actual measurements. We show that recently popularized sparse regularizations $[10,11]$ improve the situation by removing the need for the expert guess. The correct regularization is not the only ingredient though, as we explain in the sequel.

\section{PRIOR APPROACHES TO FUKUSHIMA PROBLEM}

We seek to reconstruct the temporal variation of the intensity of the radioactive release. The time of the accident being known, we might focus on estimating the amount of material released in this specific time interval. But ideally, we should be able to blindly reconstruct the timing of releases. This is valuable since it would permit us to detect radioactive material releases that otherwise went unnoticed. A blind reconstruction would also strongly suggest that the correct temporal reconstruction does not result from the a priori guess.

In the discrete formulation (2), $\mathbf{y}$ contains the concentration measurements of radioactive Xenon-isotopes in air at stations from the Comprehensive Nuclear-Test-Ban Treaty Organization (CTBTO) monitoring network. This network comprises 25 stations equipped with very sensitive radioactive Xenon detectors. The detectors typically have three stages. Two to concentrate and purify the gas sample, and the third one to measure the activity of the final gas sample. The duration of the sampling and purification process limits the number of samples per day to between one and three, depending on the detector model [12]. These systems allow to measure ${ }^{133} \mathrm{Xe}$ to an accuracy of $0.1 \mathrm{mBq} \mathrm{m}^{-3}$ [4]. Measurements from 15 CTBTO and 2 non-CTBTO stations are eventually used to solve the inverse problem. The locations of these stations are shown in Figure 2(C). Interestingly, measurements from the CTBTO station located in Japan could not be used because the levels of Xenon were over the highest detectable level of the system, saturating the detectors. All the measurements were corrected for radioactive decay. For additional information on the pre-processing of the data see [4].

On the right hand side in (2), the source term $x$ contains the rate of release of Xenon in $\mathrm{Bq} \mathrm{s}^{-1}$, at the location of Fukushima Daiichi NPP between March 10 and March 16. Temporal resolution is three hours and three different ejection heights are considered. Different heights, $0-50 \mathrm{~m}, 50-300 \mathrm{~m}$, and 300-1000 m, are necessary since the atmospheric transport of particles depends substantially on the altitude of the source [4]. Finally, the model matrix A describes every measurement as a linear combination of source terms. The coefficients of $\mathbf{A}$ are computed using FLEXPART. The number of measurements is relatively low, so the coarse discretization is necessary. The sensitivity matrix has dimensions $858 \times 120$. Since it is an overdetermined system, the first idea is to find the least-squares solution,

$$
\widehat{\mathbf{x}}=\arg \min \|\mathbf{A} \mathbf{x}-\mathbf{y}\|_{2}^{2} .
$$

Minimization (3) has a closed form solution given in terms of the Moore-Penrose pseudoinverse of $\mathbf{A}, \widehat{\mathbf{x}}=\mathbf{A}^{\dagger} \mathbf{y}=\left(\mathbf{A}^{\top} \mathbf{A}\right)^{-1} \mathbf{A}^{\top}$. However, applying the pseudoinverse does not do a good job. This happens for 2 reasons: 1) the matrix has a huge condition number and 2) the matrix likely exhibits a sizeable model mismatch, and there is noise in the measurements (although negligible in comparison with the model mismatch). We might attempt to fix the conditioning issues by the Tikhonov regularization, but the result would still be unsatisfactory. We need a good model for the source term, and assuming that it minimizes the Euclidean norm of the measurements has no justification.

The dispersion modeling community developed specific methods to cope with these nuisances. Typically, they aid the estimation by forming an expert-knowledge-based guess of the result. In the Fukushima case this comprises knowledge about when the accidents took place, what specific parts of the power plant were affected at what times, and the general expert knowledge about the nuclear power plant technology. The minimization then involves terms favoring the a priori solution, and an additional smoothing term with a second discrete derivative. If we have an estimate of uncertainties of different components in the guess, and similarly for observations (since they are obtained by techniques of different quality), we may also include these in the solution. Denoting by $\mathbf{x}_{a}$ the initial guess, 
this leads to the following convex program,

$$
\begin{aligned}
\widehat{\mathbf{x}}=\arg \min \| & \mathbf{W}_{y}(\mathbf{A x}-\mathbf{y}) \|_{2}^{2} \\
& +\lambda\left\|\mathbf{W}_{a}\left(\mathbf{x}-\mathbf{x}_{a}\right)\right\|_{2}^{2}+\varepsilon\left\|\mathbf{D}_{2}\left(\mathbf{x}-\mathbf{x}_{a}\right)\right\|_{2}^{2},
\end{aligned}
$$

where $\mathbf{D}_{2}$ is the discrete second derivative, $\mathbf{W}_{y}^{\top} \mathbf{W}_{y}=\mathbf{C}_{y}^{-1}$ and $\mathbf{W}_{a}^{\top} \mathbf{W}_{a}=\mathbf{C}_{x}^{-1}$, if $\mathbf{C}_{y}$ and $\mathbf{C}_{x}$ are the covariance matrices of the observations and of the initial guess.

Estimation using (4) was performed in [4]. The authors obtain good results with this technique but the caveat is that it is difficult to assess it, since it is very close to the a priori solution (admitting that the initial guess may be very good). To exemplify the point, in Figure 1(A) we plot the solution to (4) with the initial guess used by the authors. The corresponding measurements were generated by plugging their initial guess into the model, and then adding noise $(\mathrm{SNR}=10 \mathrm{~dB})$ to simulate the model mismatch. Now we relocate a peak and rerun the experiment. The result is shown in Figure 1(B). The solution now perfectly follows the relocated peak, and the old peak appears smoothed out. In conclusion, the method is useful, but very sensitive to the initial solution. If we account for this through "small" $\mathbf{W}_{a}$ (corresponding to large uncertainties), then we drift towards the Tikhonov regularization, since "small" $\mathbf{W}_{a}$ makes the a priori knowledge term small or negligible. But Tikhonov was shown not to perform well in [4]. In summary, what we seek is a method to correctly detect the emission times without the a priori expert knowledge. It would be wrong to just dismiss the available expert estimate, but a good method must give plausible results even without it.

\section{INGREDIENTS OF A GOOD SOLUTION}

We show that a successful solution to Problem 1 comprises three ingredients. One of the critical steps is data pre-processing. This is often encountered in the machine learning world. We identified the following key ingredients,

1) Choosing the proper regularization,

2) Cleaning the matrix,

3) Incorporating the natural constraints on the solution (such as positivity).

1) Proper regularization. Xenon ejection waveforms (estimates) in Figure 1(A) reveal that it was released in short bursts (some materials like Cesium are released during more extended periods), The temporal variation of the Xenon emission exhibits several peaks and many small elements. Therefore, a proper regularizations should favor signals with many zeros and a few large elements. This is in contrast with what is known about the regularization based on $\ell_{2}$ norms, such as Tikhonov. These favor many small/moderate elements. We should find the solution yielding something close to the observed measurements, but with as many zero elements as possible. Since minimizing the number of non-zeros is not tractable, we use the standard relaxation based on the $\ell_{1}$ norm. The solution is termed basis pursuit denoising [13],

$$
\widehat{\mathbf{x}}=\arg \min \|\mathbf{A x}-\mathbf{y}\|_{2}^{2}+\lambda\|\mathbf{x}\|_{1}
$$

2) Cleaning the matrix. Figure 2(A) shows the matrix used in [4]. We notice many very small elements (note that the color bar is in logarithmic scale). That is, many matrix rows have zero or negligible norms, meaning that these sensors do not contribute to the result w.r.t. rows with larger norms. Same figure shows the corresponding distribution of the measuring stations around the world. The smallnorm rows deteriorate the solution of the inverse problems. This can

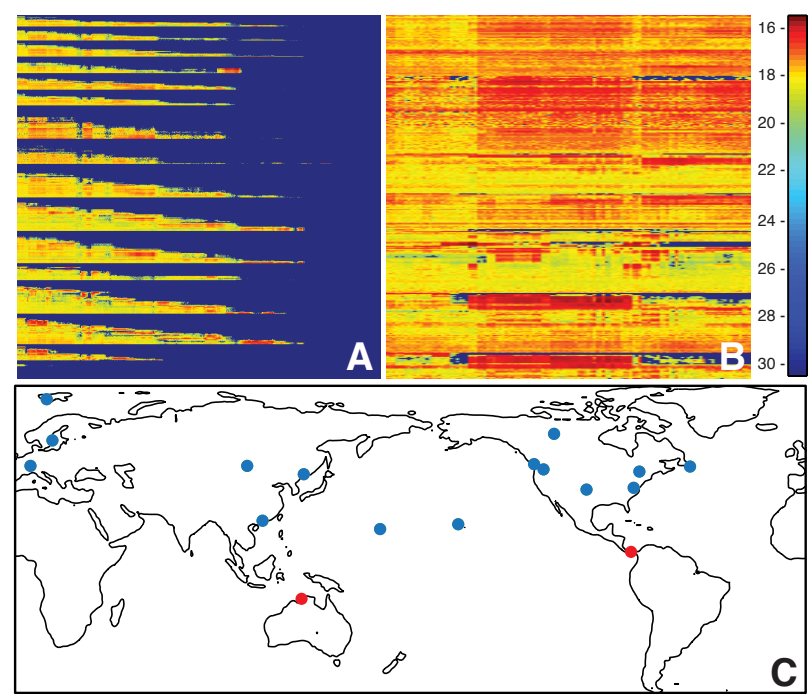

Fig. 2. Matrix (A) before and (B) after cleaning. (C) Measurement stations plotted on the world map. The red dots correspond to measurements stations that are completely left out of the consideration after cleaning the matrix.

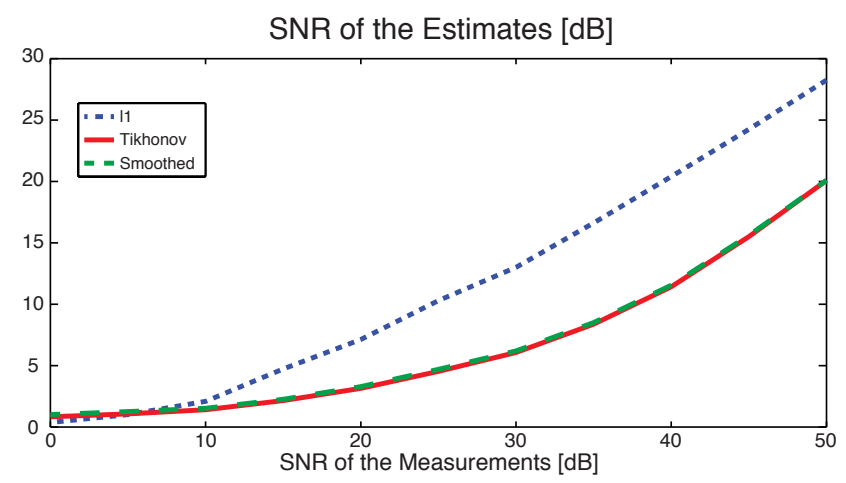

Fig. 3. SNR of the recovered source term as a function of the SNR of the synthetic measurement obtained by the forward-model matrix and an a priori source term from [4], with added Gaussian noise.

be interpreted through a very unfavorable condition number of the model matrix A After removing rows with very small norms, we are left with the matrix shown in Figure 2(B). It is evident from the color map that the remaining rows have much narrower dynamics. Even so, the elements still differ by several orders of magnitude.

It is interesting to see that cleaning the matrix removes two southernmost stations, as indicated in Figure 2. The CTBTO website features a fascinating video explaining that the equator was acting as a dividing line between the northern and the southern hemisphere air masses [14]. This means that the measurement stations located on the southern hemisphere could not sense the cloud since the could not pass the equator. Eventually the material got transported to the southern hemisphere but in very small amounts.

3) Incorporating the natural constraints. The ingredients 1) and 2) already give a reasonable result. We can find a regularization parameter $\lambda$ in the program (5) that gives $\widehat{\mathbf{x}}$ with correct release times. But changing the regularization parameter yields a very different solution and it is not clear how to properly choose $\lambda$. Furthermore, both solutions shows unrealistic negative values. An obvious solution is to enforce the non-negativity constraint to get a new convex 


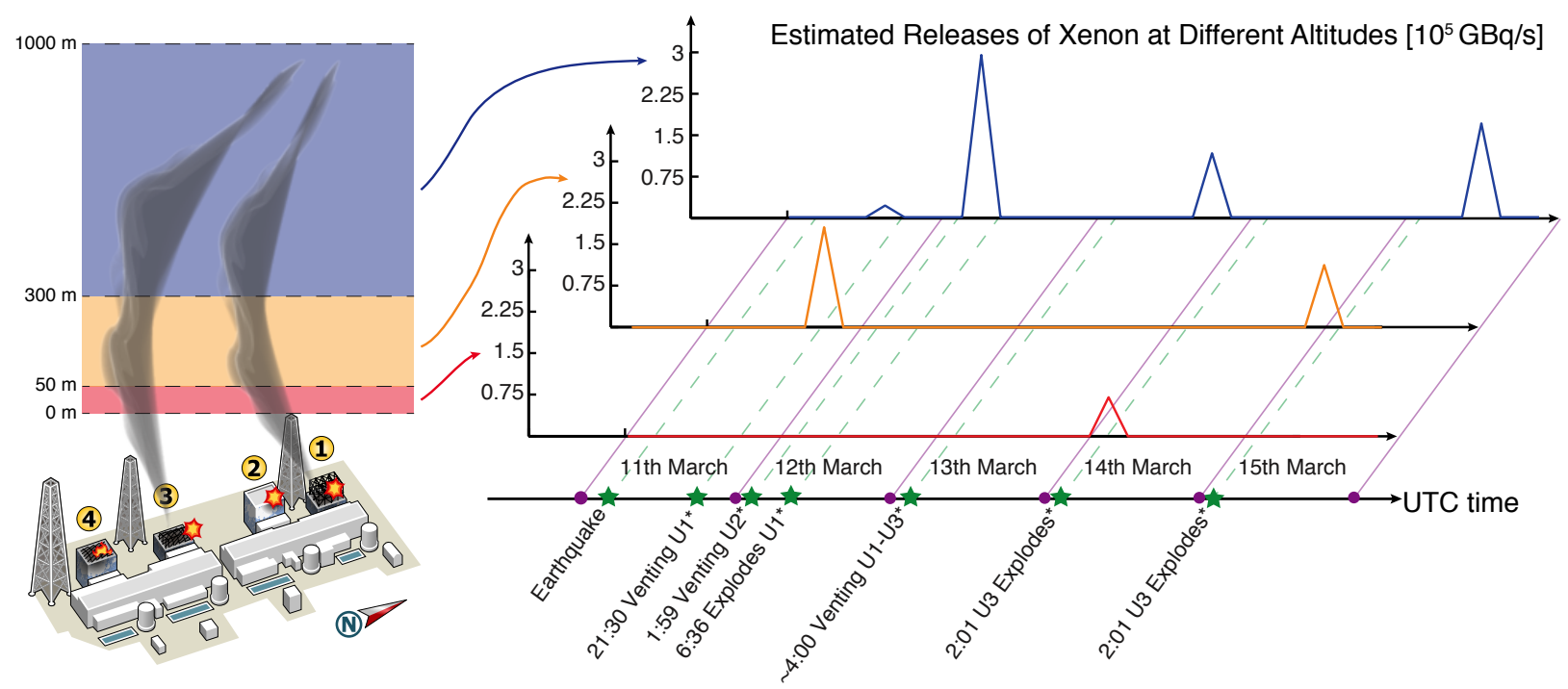

Fig. 4. Reconstruction of the emission rates using the proposed algorithm. Reconstructed rates are shown for the three considered heights. Note that, the largest peaks are in correspondence of the venting events, considered to be the main responsible of the Xenon releases. The timeline indicates events at the Fukushima NPP in the temporal window of interest and is built from numerous sources, listed on the Wikipedia page for the Fukushima I Nuclear accident.

program,

$$
\widehat{\mathbf{x}}=\arg \min \|\mathbf{A} \mathbf{x}-\mathbf{y}\|_{2}^{2}+\lambda\|\mathbf{x}\|_{1} \text { subject to } \mathbf{x} \succeq 0 .
$$

This seems to stabilize the solution. We observe that the solution is stable across a very wide range of lambdas.

Finally, a word on the choice of the regularization parameter. A possible way to determine $\lambda$, is to compute the $\ell_{1}$ and $\ell_{2}$ norms in (6) for the prior solution and estimate the regularization term based on the ratio of these norms. Since the solution, and in particular the support, varies little with $\lambda$, this is justified in the sense that it will not bias the solution towards the initial guess.

\subsection{Sensitivity test}

Another way to assess the robustness of different methods is to measure how sensitive they are with respect to errors in measurements. To this end we simulated the reconstruction by the Tikhonov-regularized program, regularization with the second derivative smoothing, and the $\ell_{1}$-based reconstruction (without the positivity constraint) while adding different amounts of noise to measurements. Measurements were generated using the real matrix and the initial Xenon guess used in [4]. The $\ell_{1}$-regularized approach performs the best among the tested methods.

\section{EXPERIMENTS WITH REAL DATA}

We apply the proposed solution (6) to real data with the cleaned matrix. The measurements and the matrix that we use are the ones described in Section 3. We repeat that this experiment is completely blind, not involving any prior information about the source except that it is sparse and positive.

Before giving the results, let us try to argue why a successful estimation would be surprising: If you look at tracer experiment results in [7], one can see that even though there is correlation between the measured and numerically predicted values, the variability is considerable - often orders of magnitude. We would expect all conventional algorithms to have great trouble estimating anything reasonable (think compressed sensing reconstruction with matrix elements orders of magnitudes wrong). The estimation is based on measurements taken on different continents, thousands of miles away from Fukushima. In spite of this, the combination of the three described ingredients yields correct blind estimates of the accident times, and the correct order of magnitude for the ejected amounts (as verified against the solution in [4] and amounts estimated by other parties [15]). The results are illustrated with respect to the timeline of the Fukushima accident for all three heights in Figure 4. We see that the algorithm correctly estimates major ventings, and in fact to a good extent matches the a priori solution mentioned earlier, but being completely blind. The waveforms also exhibit some peculiar details. For example, there is a peak just after the earthquake, but before official explosion. Could it be that already at that moment some radioactive Xenon was released into the atmosphere?

\section{CONCLUSION}

We have successfully estimated the times and quantities of radioactive Xenon emissions from the Fukushima power plant following the March 2011 earthquake. The complete solution involves sparsity promoting regularizations, as the observed Xenon emission waveforms consist of a few peaks and many very small elements. We have shown that standard approaches from the dispersion modeling community lack of stability w.r.t. their a priori guess. Future work involves testing of the sensitivity of our approach to model mismatch, revisiting the results of the tracer-experiment verification of FLEXPART, and repeating the experiment for Cesium.

\section{ACKNOWLEDGMENTS}

We thank all the researchers who produced the CTBTO measurement data, K. Ungar and I. Hoffman from Sydney for the Xenon data, H. Miley from Richland for the Xe data. We gratefully acknowledge Mikhail Kanevski for the fruitful discussions. 


\section{REFERENCES}

[1] International Atomic Energy Agency, The International $\mathrm{Nu}$ clear and Radiological Event Scale, International Atomic Energy Agency, Vienna (Austria), 2008.

[2] R. E. Rowland, Radium in humans: a review of US studies, Argonne National Lab., IL (United States), 1994.

[3] J. Pudykiewicz, "Simulation of the Chernobyl dispersion with a 3D hemispheric tracer model," Tellus B, vol. 41, no. 4, pp. 391-412, 1989.

[4] A. Stohl, P. Seibert, G. Wotawa, D. Arnold, J. F. Burkhart, S. Eckhardt, C. Tapia, A. Vargas, and T. J. Yasunari, "Xenon133 and caesium-137 releases into the atmosphere from the Fukushima Dai-ichi nuclear power plant: determination of the source term, atmospheric dispersion, and deposition," Atmos. Chem. Phys., vol. 12, no. 5, pp. 2313-2343, 2012.

[5] M. Chino, H. Nakayama, H. Nagai, H. Terada, G. Katata, and H. Yamazawa, "Preliminary estimation of release amounts of $131 \mathrm{I}$ and $137 \mathrm{Cs}$ accidentally discharged from the Fukushima Daiichi nuclear power plant into the atmosphere," J. Nucl. Sci. Technol., vol. 48, no. 7, pp. 1129-1134, 2011.

[6] A. Stohl, C. Forster, A. Frank, P. Seibert, and G. Wotawa, "Technical note: The Lagrangian particle dispersion model FLEXPART version 6.2," Atmos. Chem. Phys. Discuss., vol. 5, no. 4, pp. 4739-4799, 2005.

[7] A. Stohl, M. Hittenberger, and G. Wotawa, "Validation of the Lagrangian particle dispersion model FLEXPART against large-scale tracer experiment data," Atmos. Environ., vol. 32, no. 24, pp. 4245-4264, 1998.
[8] A. Ribes and F. Schmitt, "Linear inverse problems in imaging," IEEE Signal Process. Mag., vol. 25, no. 4, pp. 84-99, 2008.

[9] P. Seibert, "Inverse modelling of sulfur emissions in Europe based on trajectories," Geoph. Monog. Series, vol. 114, pp. 147-154, 2000.

[10] A. M. Bruckstein, D. L. Donoho, and M. Elad, "From Sparse Solutions of Systems of Equations to Sparse Modeling of Signals and Images," SIAM Review, vol. 51, no. 1, pp. 34-81, 2009.

[11] E. J. Candes and M. B. Wakin, "An Introduction To Compressive Sampling," IEEE Signal. Proc. Mag., vol. 25, no. 2, pp. 21-30, 2008.

[12] B. Wernsperger and C. Schlosser, "Noble gas monitoring within the international monitoring system of the comprehensive nuclear test-ban treaty," Radiat. Phys. Chem., vol. 71, no. 3-4, pp. 775-779, Oct. 2004.

[13] S. S. Chen, D. L. Donoho, and M. A. Saunders, "Atomic decomposition by basis pursuit," SIAM J. Sci. Comput., vol. 20, no. 1, pp. 33-61, 1998.

[14] CTBTO Preparatory Commision, The 11 March Japan Disaster, http://www.ctbto.org/verification-regime/the-11-marchjapan-disaster/, 2011.

[15] T. Y. Tanaka, T. Maki, M. Kajino, and T. T. Sekiyama, "Inverse estimation of the emission of radioactive materials from Fukushima ," Tech. Rep., Meteorological Research Institute, Japan Meteorological Agency, 2012. 